\title{
O efeito da autocorrelação no planejamento das cartas de controle de $\overline{\mathrm{X}}$ e EWMA
}

\author{
The effect of the autocorrelation on the design of \\ the $\bar{X}$ and EWMA control charts
}

\author{
Roberto Campos Leoni ${ }^{1}$ \\ Antônio Fernando Branco Costa ${ }^{1}$ \\ Marcela Aparecida Guerreiro Machado'
}

\begin{abstract}
Resumo: No planejamento dos gráficos de controle destinados ao monitoramento da média do processo, assume-se que esta permanece fixa em seu valor-alvo até a ocorrência de uma causa especial, que a desloca. Em muitos processos, contudo, é mais razoável supor que a média oscila mesmo na ausência de causas especiais. Para descrever este comportamento oscilatório, tem-se utilizado o modelo AR (1). Quando esta oscilação é grande, o melhor desempenho do gráfico de $\bar{X}$ é obtido com amostras unitárias. O mesmo não se observa com a carta de EWMA (exceto quando o parâmetro de ponderação $\lambda$ é próximo de um); os melhores desempenhos são obtidos com a adoção de amostras de tamanho $n>1$ e $\lambda$ pequeno, mesmo quando o objetivo é a detecção rápida de grandes deslocamentos da média. Neste estudo, tem-se utilizado como medida de desempenho o TES - tempo médio entre a ocorrência de uma mudança na posição em torno da qual a média oscila e sua sinalização pelo gráfico de controle. Quando a média do processo oscila, o TES passa a ser uma função do número esperado de visitas aos estados transientes de uma cadeia de Markov.
\end{abstract}

Palavras-chave: Gráfico de $\bar{X}$. Gráfico de controle de EWMA. Autocorrelação. Controle estatístico de processos. Controle estatístico de qualidade. Cadeias de Markov.

\begin{abstract}
The design of the control charts for the process mean assumes that this parameter remains fixed on its target value until the occurrence of a special cause that moves it. However, in many cases, it is more reasonable to assume that the mean wanders even in the absence of special causes. The AR(1) model has been considered to describe this wandering behavior. When the wandering behavior is responsible for significant proportion of data variability, the best performance of the $\bar{X}$ chart is obtained with samples of size one $(n=1)$. The same is not true for the EWMA control chart (except when the smoothing parameter $\lambda$ is very close to one); its best performance is achieved with the adoption of $n>1$ and small $\lambda$, even when the focus is to easily detect significant changes in the process mean position. In this study, the ATS, the average time between the occurrence of a change in the process mean position and the signal, was used as a performance measure. When the process mean wanders, the ATS becomes a function of the expected number the transient states of a Markov chain are visited
\end{abstract}

Keywords: $\bar{X}$ chart. EWMA control chart. Autocorrelation. Statistical process control. Statistical quality control. Markov chains.

\section{Introdução}

Os gráficos de controle são "ferramentas estatísticas" propostas por Shewhart (1931) para o monitoramento de processos; basicamente, detectam causas especiais que alteram a sua média e/ou a variância. O gráfico de $\bar{X}$ sinaliza mudanças na média $\mu$ do processo com base em observações de $X$, uma característica de qualidade mensurável; por exemplo, o diâmetro de um eixo ou a quantidade de refrigerante em uma garrafa. Em geral, assume-se que, desde o início da produção até o instante da ocorrência da causa especial, a média do processo permanece fixa em um valor pré-estabelecido, chamado de "valor-alvo" e a partir de então se desloca. Em certos casos, no entanto, ela exibe um comportamento oscilatório que pode estar associado a uma causa especial ou ser parte integrante da variabilidade natural do processo. No primeiro caso, basta eliminar a causa especial, já no segundo, não se deve fazer vistas grossas à oscilação, pois só com um modelo que prevê com precisão o comportamento oscilatório da média é

\footnotetext{
${ }^{1}$ Departamento de Produção, Faculdade de Engenharia de Guaratinguetá - FEG, Universidade Estadual Paulista

"Júlio de Mesquita Filho" - UNESP, Av. Dr. Ariberto Pereira da Cunha, 333, CEP 12516-410, Guaratinguetá, SP, Brasil,

e-mail: rcleoni@yahoo.com.br; fbranco@feg.unesp.br; marcela@feg.unesp.br
}

Recebido em 19/9/2010 — Aceito em 14/8/2012

Suporte financeiro: CAPES, CNPq e FAPESP. 
possível estimar a taxa de alarmes falsos e o poder de detecção do gráfico de controle.

Não são poucos os processos em que a média da distribuição das observações de $X$ oscila. Produções por bateladas ou lotes são cenários típicos em que isto ocorre. Nestes processos, unidades de um mesmo lote apresentam pouca variação, contudo a oscilação da média gera uma variabilidade adicional entre unidades de diferentes lotes, Costa, Epprecht e Carpinetti (2005). A seção 6 apresenta dois processos em que a média oscila; um de fabricação de rolhas metálicas (VASCONCELOS; ALEXANDRE; FREITAS, 2010) e outro de usinagem (CLARO, 2008).

Reynolds Junior, Arnold e Baik (1996) foram os pioneiros a investigar o efeito da oscilação da média no desempenho dos gráficos de controle. O NMA - número médio de amostras até o sinal (veja COSTA; EPPRECHT; CARPINETTI (2005) para maiores detalhes) - tem sido utilizado como parâmetro de medida de desempenho. Para obter o NMA, Reynolds Junior, Arnold e Baik (1996) e todos os demais pesquisadores que se dedicaram a investigar o efeito oscilatório da média no desempenho dos gráficos de controle, como Van Brackle III e Reynolds Junior (1997), Lu e Reynolds Junior (1999a, b, 2001), Lin e Chou (2008), Zou, Wang e Tsung (2008) e Lin (2009), recorreram a um procedimento bastante complexo, envolvendo cadeias de Markov e quadratura numérica para a solução de equações integrais. Neste artigo, apresentamos um procedimento mais simples para obtenção das propriedades dos gráficos de controle, isto é, se os estados transientes da cadeia são adequadamente definidos, o NMA é simplesmente uma função do número esperado de vezes em que eles são visitados, antes do estado absorvente ser alcançado.

O planejamento de um gráfico de controle de $\bar{X}$ consiste na determinação de seus parâmetros de construção, $n, h$ e $L$, respectivamente, o tamanho das amostras, o intervalo entre retirada de amostras e o fator de abertura dos limites de controle. Uma vez definido o mínimo TMAF - tempo médio entre alarmes falsos - e a taxa máxima de inspeção $\mathrm{TI}=n / h$, é possível descobrir a combinação $(n, h, L)$ que minimiza o TES - tempo esperado para a detecção de uma causa especial que desloca a média do processo de seu valor-alvo. Como o NMA é o número médio de amostras que o gráfico de controle requer para sinalizar, segue que, multiplicado o NMA pelo $h$, tem-se o tempo médio entre o instante de retirada da última amostra, que antecede o deslocamento da média, e o instante em que o gráfico de controle sinaliza o desajuste. Por simplicidade e sem perda de precisão, supõe-se, na obtenção do TES, que a média do processo deixa seu valor-alvo em um ponto médio no tempo entre a retirada de duas amostras consecutivas, portanto TES $=\mathrm{NMA}^{*} h-h / 2$. Costa,
Epprecht e Carpinetti (2005) introduziram esta metodologia de obtenção dos valores ótimos de $(n, h, L)$. Neste artigo aplica-se esta metodologia para o caso em que a média do processo oscila, e então, investiga-se o quanto o efeito oscilatório da média afeta os valores ótimos de $(n, h, L)$, bem como o desempenho do gráfico de $\bar{X}$ e de EWMA.

A hipótese adotada neste estudo é a mesma adotada por Reynolds Junior, Arnold e Baik (1996), Lu e Reynolds Junior (1999a, b, 2001), Lin e Chou (2008), Zou, Wang e Tsung (2008) e Lin (2009), isto é, quando em controle a média do processo oscila em torno de seu valor-alvo, já no período fora de controle ela permanece oscilando, porém não mais em torno de seu valor-alvo. Entretanto, em todos estes estudos, foi adotada uma relação de dependência entre a magnitude do movimento oscilatório da média e a magnitude do seu deslocamento provocado pela causa especial, isto é, quanto maior a amplitude do movimento oscilatório maior o deslocamento da média. Não há razões que justifiquem essa dependência. É mais realístico tratar o deslocamento da média e o seu comportamento oscilatório como dois eventos independentes. Se este não for o caso, então as regras de dependência devem ser mais bem estudadas. $\mathrm{Na}$ seção 3 , este assunto será discutido à luz do modelo proposto.

Na seção 2, é introduzido o modelo autorregressivo de primeira ordem, AR(1), que descreve o comportamento oscilatório da média do processo. Na seção 3, apresenta-se o desempenho do gráfico de $\bar{X}$ e, na seção 4, o do gráfico EWMA. Na seção 5, obtêm-se os valores ótimos de $(n, h, L)$ que minimizam o TES dos gráficos de $\bar{X}$ e de EWMA utilizados no monitoramento de um processo cuja média oscila; e, na seção 6, descrevem-se aplicações em que as observações de $X$ não são independentes, em consequência do comportamento oscilatório da média do processo. No Apêndice, descreve-se a cadeia de Markov utilizada na determinação do NMA do gráfico de $\bar{X}$.

\section{Definição do modelo que descreve as observações de $X$}

$O$ modelo que se ajusta às observações de $X$ tem duas componentes aleatórias: uma associada ao comportamento oscilatório da média do processo, observada entre subgrupos racionais, razoavelmente espaçados no tempo; e outra associada à variabilidade natural entre observações de $X$ pertencentes a um mesmo subgrupo racional. Quando os gráficos de $\bar{X}$ ou EWMA estão em uso, a cada intervalo de tempo $h$, retira-se do processo uma amostra de tamanho $n$, e então são registrados os $n$ valores de $X$ de uma característica de qualidade mensurável. Se $\mu_{k}$ é a média do processo no instante em que a $k$-ésima 
amostra é formada e $e_{k i}$ é o erro aleatório associado ao i-ésimo item da $k$-ésima amostra, fruto da imprecisão do instrumento de medida e da variabilidade natural do processo, então para $i=1,2, \ldots, n$ e $k=1,2, \ldots$,

$$
X_{k i}=\mu_{k}+e_{k i}
$$

Em geral, assume-se que $e_{k i}$ é uma variável aleatória com distribuição normal de média zero e desvio padrão 1 , isto é, $e_{k i} \sim \mathrm{N}(0 ; 1)$. Neste artigo, estamos interessados em conhecer o efeito do comportamento oscilatório da média do processo no desempenho do gráfico de $\bar{X}$ e de EWMA. Este comportamento oscilatório é descrito por um modelo autorregressivo de primeira ordem $\mathrm{AR}(1)$,

$$
\mu_{k}=\left(1-\phi^{h}\right) \xi+\phi^{h} \mu_{k-1}+\alpha_{k}
$$

em que $\left|\phi^{h}\right|<1$ é a correlação entre $\mu_{\mathrm{k}}$ e $\mu_{\mathrm{k}-1}$, $\alpha_{k} \sim \mathrm{N}\left(0 ; \sigma_{\alpha}\right)$, e $\xi$ é o valor em torno do qual a média do processo oscila; com o processo em controle $\xi=\mu_{0}$, e com ele fora de controle $\xi=\mu_{0}+\delta \sigma_{e}$. Os erros aleatórios $e_{\mathrm{ki}}$ e $\alpha_{\mathrm{k}}$ são considerados independentes. De acordo com Reynolds Junior, Arnold e Baik (1996), se $\mu_{1} \sim \mathrm{N}\left(\xi ; \sigma_{\mu}^{2}\right)$, então

$$
\sigma_{\mu}^{2}=\sigma_{\alpha}^{2} /\left(1-\phi^{2 h}\right)
$$

De acordo com a expressão (1), $X$ tem duas componentes de variabilidade

$$
\sigma_{X}^{2}=\sigma_{\mu}^{2}+\sigma_{e}^{2}
$$

A proporção da variabilidade de $X$ atribuída à oscilação da média, $\psi=\sigma_{\mu}^{2} / \sigma_{X}^{2}$, tem sido utilizada como medida do grau de oscilação.

Quando $\mathrm{n}>1$, da expressão (1) decorre que $\bar{X}_{k}=\mu_{k}+\bar{e}_{k}$, com $\bar{e}_{k}=\sum_{i=1}^{n} e_{k i} / n$. A variância de $\bar{X}_{k}$ é então igual a $\sigma_{\bar{X}}^{2}=\sigma_{\mu}^{2}+\sigma_{e}^{2} / n$. Da expressão (4) e da definição de $\psi$ segue que:

$$
\sigma_{\bar{X}}=\frac{\sigma_{e}}{\sqrt{n}} \sqrt{1+\frac{n \psi}{1-\psi}}=\frac{\sigma_{e}}{\sqrt{n}} f(n, \psi)
$$

Portanto, os limites de controle (LC) são dados por: $\mu_{0} \pm L^{*} \sigma_{e} / \sqrt{n}$, em que $L^{*}=f(n, \psi) L$. Quando a média do processo não oscila, a expressão (5) se reduz a $\sigma_{\bar{X}}=\sigma_{e} / \sqrt{n}$, portanto $f(n, \psi)$ mede o quanto os limites de controle $\left(\mu_{0} \pm L \sigma_{e} / \sqrt{n}\right)$ devem ser alargados para compensar o comportamento oscilatório da média do processo. A Tabela 1 apresenta para diversos valores de $n$ e de $\psi$ o fator de abertura adicional $f(n, \psi)$ dos limites de controle, resultante do movimento oscilatório da média. Por exemplo, um $f(n, \psi)=1,3$ significa que os limites de controle alargaram-se em $30 \%$.
De acordo com as expressões (2) e (3), à medida em que $h$ aumenta, $\sigma_{\alpha}^{2}(\mathrm{~h})$ também aumenta, contudo a correlação entre $\mu_{\mathrm{k}} \mathrm{e} \mu_{\mathrm{k}-1}$, dada por $\phi^{h}$, diminui, de tal sorte que a variância $\sigma_{\alpha}^{2}$, parâmetro do movimento oscilatório da média, permanece independente de $h$. Em outras palavras, em intervalos $h$ mais espaçados, o valor da variável $\mu_{\mathrm{k}}$ depende mais do movimento oscilatório e menos do valor de $\mu_{\mathrm{k}-1}$, contudo sua variância é sempre constante e igual a $\sigma_{\alpha}^{2}$, para todo e qualquer valor de $h$. A Tabela 2 apresenta, para $\sigma_{\alpha}^{2}=5,2632$ e diferentes $h$, os valores de $\phi^{h}$ e de $\sigma_{\alpha}^{2}$.

O termo "autocorrelação" será utilizado no restante deste artigo com o seguinte significado: os valores da média do processo seguem o modelo descrito em (2).

\section{Efeito da autocorrelação no desempenho do Gráfico de $\bar{X}$}

O NMA - número médio de amostra até o sinal - tem sido adotado como medida de desempenho do gráfico controle. Quando as observações $X$ são independentes, o NMA é simplesmente o inverso do poder de detecção do gráfico de controle, isto é, $\mathrm{NMA}=1 / p . \mathrm{O}$ deslocamento da média do processo é, em geral, expresso em unidades $\delta$ do desvio padrão de $X$. Para uma amostra de tamanho $n$ e

Tabela 1. Valores do fator de abertura adicional $f(n, \psi)$.

\begin{tabular}{ccc}
\hline $\mathbf{n}$ & $\psi$ & $\boldsymbol{f}(\boldsymbol{n}, \boldsymbol{\psi})$ \\
\hline \multirow{3}{*}{1} & 0,1 & 1,05 \\
& 0,5 & 1,41 \\
& 0,9 & 3,16 \\
\hline \multirow{3}{*}{4} & 0,1 & 1,20 \\
& 0,5 & 2,24 \\
& 0,9 & 6,08 \\
\hline \multirow{2}{*}{20} & 0,1 & 1,80 \\
& 0,5 & 4,58 \\
& 0,9 & 13,45 \\
\hline
\end{tabular}

Tabela 2. Valores de $\phi^{h}$ e $\sigma_{\alpha}^{2}$.

\begin{tabular}{ccc}
\hline $\boldsymbol{h}$ & $\phi^{h}$ & $\sigma_{\alpha}^{2}(\mathbf{h})$ \\
\hline 1 & 0,9000 & 1,0000 \\
4 & 0,6561 & 2,9975 \\
8 & 0,4305 & 4,2879 \\
12 & 0,2824 & 4,8433 \\
16 & 0,1853 & 5,0824 \\
20 & 0,1216 & 5,1854 \\
30 & 0,0424 & 5,2537 \\
40 & 0,0148 & 5,2620 \\
50 & 0,0052 & 5,2630 \\
100 & 0,0000 & 5,2632 \\
\hline
\end{tabular}


um fator de abertura dos limites de controle $L$, $p=\Phi(-L+\delta \sqrt{n})-\Phi(-L-\delta \sqrt{n})$. A obtenção do NMA deixa de ser simples quando a média do processo oscila. Reynolds Junior, Arnold e Baik (1996) recorreram a um procedimento bastante complexo, envolvendo cadeias de Markov e métodos de equação integral com quadratura. Um procedimento mais simples permite obter o NMA direto de uma cadeia de Markov. Isto é, se os estados transientes da cadeia são adequadamente definidos, o NMA é simplesmente uma função do número esperado de vezes em que eles são visitados, antes do estado absorvente ser alcançado (ver Apêndice). Em Reynolds Junior, Arnold e Baik (1996), o deslocamento da média é expresso em unidades $\delta$ do desvio padrão de $X$. De acordo com as expressões (3) e (4), no caso em que a média do processo oscila, o desvio padrão de $X$ tem duas componentes de variação, uma associada ao movimento oscilatório da média $-\sigma_{\mu}$ e outra associada à variação das medidas de $X$ dentro das amostras $-\sigma_{\mathrm{e}}$. Portanto, a magnitude do deslocamento da média do processo, expressa em unidades $\delta$ do desvio padrão de $X$, depende de $\sigma_{\mu}$. Em dois processos, um em que a média não oscila, isto é $\sigma_{\mu}=0$, e outro em que ela oscila muito, isto é $\sigma_{\mu}=0,8$, a causa especial atua de forma diferente. No caso em que a média não oscila, a magnitude do deslocamento é de $\delta$ (supondo $\sigma_{\mathrm{e}}=1,00$ ); já no processo em que a média oscila, o deslocamento é $28 \%$ maior $(\sqrt{0,64+1,00} \delta)$. Não há evidências para assumir que o deslocamento da média do processo é maior nos casos em que ela oscila, e muito menos que haja uma correlação entre a magnitude do deslocamento da média $\left(\delta \sqrt{\sigma_{\mu}^{2}+\sigma_{e}^{2}}\right)$ e a variância de seu movimento oscilatório $\left(\sigma_{\alpha}^{2}\right)$. Assim, uma forma mais lógica de se analisar o efeito da autocorrelação no desempenho do gráfico de controle consiste em expressar o deslocamento da média do processo sempre em unidades $\delta$ da variação das medidas de $X$ dentro da amostra $-\sigma_{e}$. As Tabelas 3 e 4 apresentam os NMAs para diferentes combinações de $\delta, \phi$ e $\psi$, e, para efeito de comparação, deslocamentos da média $\delta \sigma_{\mathrm{e}}$ e $\delta \sigma_{\mathrm{x}}$.

Tabela 3. NMA para o gráfico de $\bar{X}$ - deslocamentos de $\delta \sigma_{\mathrm{x}}$.

\begin{tabular}{|c|c|c|c|c|c|c|c|c|}
\hline \multirow[b]{4}{*}{$\mathbf{L}$} & \multicolumn{4}{|c|}{$\mathbf{n}=\mathbf{1}$} & \multicolumn{4}{|c|}{$n=4$} \\
\hline & \multirow[t]{2}{*}{$\phi=0,0$} & \multicolumn{3}{|c|}{$\phi=+0,8$} & \multirow[t]{2}{*}{$\phi=0,0$} & \multicolumn{3}{|c|}{$\phi=+0,8$} \\
\hline & & $\psi=\mathbf{0 , 1}$ & $\psi=\mathbf{0 , 5}$ & $\psi=\mathbf{0 , 9}$ & & $\psi=\mathbf{0 , 1}$ & $\psi=\mathbf{0 , 5}$ & $\psi=\mathbf{0 , 9}$ \\
\hline & 3,0000 & 2,9993 & 2,9790 & 2,9029 & 3,0000 & 2,9929 & 2,931 & 2,8758 \\
\hline$\delta$ & \multicolumn{8}{|c|}{ NMA } \\
\hline 0,0 & 370,40 & 370,40 & 370,40 & 370,40 & 370,40 & 370,40 & 370,40 & 370,40 \\
\hline 0,5 & 155,20 & 157,90 & 171,70 & 188,60 & 45,40 & 66,90 & 138,40 & 184,20 \\
\hline 1,0 & 43,90 & 46,00 & 55,80 & 67,30 & 6,60 & 12,10 & 38,90 & 64,40 \\
\hline 1,5 & 15,00 & 16,20 & 21,40 & 27,30 & 2,10 & 3,50 & 13,70 & 25,80 \\
\hline 2,0 & 6,30 & 6,90 & 9,50 & 12,50 & 1,20 & 1,50 & 5,50 & 11,60 \\
\hline 3,0 & 2,00 & 2,10 & 2,60 & 3,20 & 1,00 & 1,00 & 1,50 & 2,90 \\
\hline 4,0 & 1,20 & 1,20 & 1,20 & 1,30 & 1,00 & 1,00 & 1,00 & 1,20 \\
\hline 5,0 & 1,00 & 1,00 & 1,00 & 1,00 & 1,00 & 1,00 & 1,00 & 1,00 \\
\hline
\end{tabular}

Tabela 4. NMA para o gráfico de $\bar{X}$ - deslocamentos de $\delta \sigma_{\mathrm{e}}$.

\begin{tabular}{|c|c|c|c|c|c|c|c|c|}
\hline \multirow[b]{4}{*}{$\mathbf{L}$} & \multicolumn{4}{|c|}{$n=1$} & \multicolumn{4}{|c|}{$n=4$} \\
\hline & \multirow[t]{2}{*}{$\phi=0,0$} & \multicolumn{3}{|c|}{$\phi=+0,8$} & \multirow[t]{2}{*}{$\phi=0,0$} & \multicolumn{3}{|c|}{$\phi=+0,8$} \\
\hline & & $\psi=\mathbf{0 , 1}$ & $\psi=\mathbf{0 , 5}$ & $\psi=\mathbf{0 , 9}$ & & $\psi=\mathbf{0 , 1}$ & $\psi=\mathbf{0 , 5}$ & $\psi=\mathbf{0 , 9}$ \\
\hline & 3,0000 & 2,9993 & 2,979 & 2,9029 & 3,0000 & 2,9929 & 2,931 & 2,8758 \\
\hline$\delta$ & \multicolumn{8}{|c|}{ NMA } \\
\hline 0,0 & 370,40 & 370,40 & 370,40 & 370,40 & 370,40 & 370,40 & 370,40 & 370,40 \\
\hline 0,5 & 155,20 & 168,50 & 238,00 & 339,40 & 45,40 & 74,20 & 206,00 & 338,10 \\
\hline 1,0 & 43,90 & 51,80 & 106,30 & 270,00 & 6,60 & 14,10 & 79,50 & 266,50 \\
\hline 1,5 & 15,00 & 18,70 & 49,20 & 198,90 & 2,10 & 4,10 & 33,90 & 194,50 \\
\hline 2,0 & 6,30 & 8,10 & 24,90 & 142,70 & 1,20 & 1,80 & 16,10 & 138,50 \\
\hline 3,0 & 2,00 & 2,40 & 7,90 & 74,40 & 1,00 & 1,00 & 4,50 & 71,40 \\
\hline 4,0 & 1,20 & 1,30 & 3,10 & 41,00 & 1,00 & 1,00 & 1,70 & 39,00 \\
\hline 5,0 & 1,00 & 1,00 & 1,60 & 23,90 & 1,00 & 1,00 & 1,10 & 22,60 \\
\hline
\end{tabular}


De acordo com as Tabelas 3 e 4, o gráfico de controle de $\bar{X}$ perde desempenho (NMA aumenta) à medida que $\psi$ aumenta. Em outras palavras, quanto maior é a parcela da variabilidade de $X$ resultante do movimento oscilatório da média do processo, em relação a sua variabilidade total, mais lento é o gráfico na sinalização de causas especiais que a faz oscilar em torno de um ponto distante de seu "valor-alvo". Deslocamentos da média de $\delta \sigma_{x}$ são maiores que de $\delta \sigma_{e}$, o que implica, naturalmente, em uma resposta mais rápida do gráfico de $\bar{X}$, pois, para deslocamentos maiores, o NMA é sempre menor. Para uma comparação justa em relação a um processo independente (processo em que a média não oscila), a Tabela 4 apresenta uma comparação adequada quando considera apenas deslocamentos de $\delta \sigma_{\mathrm{e}}$.

\section{Efeito da autocorrelação no desempenho do Gráfico EWMA}

A carta de controle de $\bar{X}$ é a mais conhecida no monitoramento de processos, porém ela só é indicada para a sinalização de grandes deslocamentos na média. O esquema concorrente denominado gráfico de controle EWMA é melhor na sinalização de pequenos desajustes, especialmente com o parâmetro de ponderação $(\lambda)$ próximo a zero (CLARO; COSTA; MACHADO, 2007).
Propõe-se, neste artigo, o uso do gráfico de EWMA com limites alargados em virtude do comportamento oscilatório da média do processo. Com o processo em controle, os limites de ação do gráfico de EWMA foram então ajustados para se ter um NMA de 370,4. Os NMAs foram obtidos por simulação; segundo Lu e Reynolds Junior (1999b), a aplicação de métodos numéricos para determinar o NMA do gráfico de controle de EWMA é computacionalmente lenta e, dependendo dos parâmetros do modelo, alguns resultados são incertos; portanto, o uso da simulação nestes casos leva a resultados mais confiáveis. As Tabelas 5 a 9 apresentam os NMAs para diferentes combinações de $\delta, \lambda$, $\phi$ e $\psi$. Nas Tabelas 5 a 9, aparecem em destaque (negrito) os valores mínimos do NMA.

De acordo com as Tabelas 4 e 5, quando a média do processo não oscila, $\mathrm{n}=1$ e $\delta \leq 2$, o gráfico EWMA sinaliza com mais rapidez se comparado com o gráfico de controle de $\bar{X}$ (ver Tabela 4) na sinalização. Por exemplo, para $\lambda=0,05, \mathrm{n}=1$ e $\delta=0,5$, o esquema EWMA requer em média 25,7 amostras (Tabela 5) para detectar a alteração na média do processo, enquanto que o gráfico de $\bar{X}$ requer 155,20 amostras (Tabela 4) para detectar a mesma alteração. Na Tabela 5, observa-se que, aumentando $\lambda$, o desempenho do esquema EWMA se assemelha

Tabela 5. NMA para o gráfico EWMA $-\psi=0 ; \mathrm{n}=1$ e 4 .

\begin{tabular}{|c|c|c|c|c|c|c|c|c|c|c|c|c|c|c|c|c|c|c|c|}
\hline $\mathbf{N}$ & & & & & 1,0 & & & & & & & & & & 4,0 & & & & \\
\hline$\delta=(\xi-$ & $\xi 0) / \sigma_{e}$ & 0,5 & 1,0 & 1,5 & 2,0 & 2,5 & 3,0 & 4,0 & 5,0 & $\delta=(\xi-$ & $\xi(0) / \sigma_{\mathrm{e}}$ & 0,5 & 1,0 & 1,5 & 2,0 & 2,5 & 3,0 & 4,0 & 5,0 \\
\hline $\mathbf{L}$ & $\lambda$ & & & & & & & & & L & $\lambda$ & & & & & & & & \\
\hline 2,490 & 0,05 & 25,7 & 10,5 & 6,7 & 4,9 & 4,0 & 3,3 & 2,6 & 2,1 & 2,485 & 0,05 & 10,5 & 4,9 & 3,3 & 2,6 & 2,1 & 1,9 & 1,5 & 1,3 \\
\hline 2,701 & 0,10 & 27,5 & 9,5 & 5,7 & 4,1 & 3,3 & 2,8 & 2,1 & 1,8 & 2,696 & 0,10 & 9,5 & 4,1 & 2,7 & 2,1 & 1,8 & 1,5 & 1,2 & 1,0 \\
\hline 2,805 & 0,15 & 31,3 & 9,4 & 5,3 & 3,8 & 2,9 & 2,5 & 1,9 & 1,6 & 2,802 & 0,15 & 9,4 & 3,8 & 2,5 & 1,9 & 1,6 & 1,4 & 1,1 & 1,0 \\
\hline 2,858 & 0,20 & 35,4 & 9,6 & 5,1 & 3,5 & 2,7 & 2,3 & 1,8 & 1,5 & 2,855 & 0,20 & 9,6 & 3,5 & 2,3 & 1,8 & 1,5 & 1,2 & 1,0 & 1,0 \\
\hline 2,895 & 0,25 & 40,5 & 10,0 & 5,1 & 3,4 & 2,6 & 2,1 & 1,6 & 1,3 & 2,896 & 0,25 & 10,1 & 3,4 & 2,1 & 1,6 & 1,3 & 1,1 & 1,0 & 1,0 \\
\hline 2,925 & 0,30 & 46,2 & 10,7 & 5,1 & 3,3 & 2,5 & 2,1 & 1,6 & 1,3 & 2,925 & 0,30 & 10,7 & 3,3 & 2,1 & 1,6 & 1,3 & 1,1 & 1,0 & 1,0 \\
\hline 2,945 & 0,35 & 52,0 & 11,6 & 5,2 & 3,3 & 2,4 & 2,0 & 1,5 & 1,2 & 2,945 & 0,35 & 11,6 & 3,3 & 2,0 & 1,5 & 1,2 & 1,1 & 1,0 & 1,0 \\
\hline 2,956 & 0,40 & 57,5 & 12,5 & 5,4 & 3,3 & 2,4 & 1,9 & 1,4 & 1,2 & 2,960 & 0,40 & 12,5 & 3,3 & 1,9 & 1,4 & 1,2 & 1,0 & 1,0 & 1,0 \\
\hline 2,976 & 0,50 & 71,3 & 15,1 & 5,9 & 3,4 & 2,4 & 1,8 & 1,3 & 1,1 & 2,980 & 0,50 & 15,1 & 3,4 & 1,8 & 1,3 & 1,1 & 1,0 & 1,0 & 1,0 \\
\hline & $(\xi-\xi$ & $0) / \sigma_{e}$ & & 0,5 & & 1,0 & & 1, & & 2,0 & & 2,5 & & 3,0 & & 4,0 & & 5 & \\
\hline $\mathbf{L}$ & & $\lambda$ & & & & & & & & & & & & & & & & & \\
\hline 3,47 & & 0,05 & & 199,7 & & 88, & & 48 & & 31,5 & & 22,9 & & 17,9 & & 12,3 & & 9 & \\
\hline 3,68 & & 0,10 & & 228,8 & & 107, & & 56 & & 34,8 & & 23,8 & & 17,6 & & 11,3 & & 8 & \\
\hline 3,74 & & 0,15 & & 251,0 & & 125, & & 66 & & 39,6 & & 26,0 & & 18,6 & & 11,2 & & 7 & \\
\hline 3,75 & & 0,20 & & 263,8 & & 140 & & 75 & & 44,6 & & 28,6 & & 20,0 & & 11,5 & & 7 & \\
\hline 3,73 & & 0,25 & & 273,6 & & 152,3 & & 83 & & 49,5 & & 31,7 & & 21,8 & & 12,0 & & 7 & \\
\hline 3,70 & & 0,30 & & 284,1 & & 163, & & 91 & & 54,8 & & 34,7 & & 23,5 & & 12,6 & & 8 & \\
\hline 3,65 & & 0,35 & & 287,9 & & 170, & & 98 & & 58,8 & & 37,4 & & 25,4 & & 13,3 & & 8 & \\
\hline 3,62 & & 0,40 & & 299,2 & & 182,8 & & 107 & & 64,6 & & 41,2 & & 27,5 & & 14,2 & & 8 & \\
\hline 3,51 & & 0,50 & & 299,5 & & 195, & & 117 & & 72,5 & & 46,2 & & 31,0 & & 15,7 & & 9 & \\
\hline
\end{tabular}


ao do gráfico de $\bar{X}$, lembrando que, $\underline{\operatorname{com}} \lambda=1$, o gráfico de EWMA é um gráfico de $\bar{X}$ (COSTA; EPPRECHT; CARPINETTI, 2005).

A autocorrelação entre os valores da média do processo, segundo o modelo descrito em (2), prejudica o desempenho dos gráficos de controle. Tem-se um aumento significativo no número médio de amostras que o gráfico de controle requer para sinalizar uma alteração na média do processo. Na Tabela 5, para $\mathrm{n}=1, \lambda=0,05$ e um deslocamento da média do processo de $\delta=0,5$, isenta de oscilação, isto é, $\sigma_{\mu}=0, o \mathrm{NMA}=25,7$, ao passo que, na presença de uma correlação moderada $(\phi=0,4)$ e uma parcela alta de variabilidade de $X$ fruto da oscilação da média $(\psi=0,9)$, o NMA = 199,7 (ver Tabela 6), ou seja, aproximadamente 7,8 vezes maior.

Quando comparado com o gráfico de $\bar{X}$, o esquema EWMA, na presença de forte oscilação da média $(\psi=0,9)$ e elevado grau de autocorrelação $(\phi=0,8)$, se mostra mais ágil na detecção de pequenos e grandes deslocamentos. Por exemplo, para $\mathrm{n}=1$ e $\delta=0,5$, o $\mathrm{NMA}=339,40$ (Tabela 4), caso esteja em uso o gráfico de $\bar{X}$, e NMA $=283,8$ (Tabela 7), caso esteja em uso o gráfico de EWMA $(\lambda=0,05)$. Quando há um grande deslocamento $(\delta=5,0)$, tem-se para o gráfico de $\bar{X}$ um NMA $=23,9$ (Tabela 4$)$, enquanto que, para o gráfico de EWMA, o NMA = 19,0 (Tabela 7). Para $n=4$ e $\delta=0,5$, o NMA $=338,10$ (Tabela 4),

Tabela 7. NMA para o gráfico de EWMA $-\psi=0,9 ; \mathrm{n}=1 ; \phi=0,8$.

\begin{tabular}{|c|c|c|c|c|c|c|c|c|c|}
\hline \multicolumn{2}{|c|}{$\delta=(\xi-\xi 0) / \sigma_{\mathbf{e}}$} & 0,5 & 1,0 & 1,5 & 2,0 & 2,5 & 3,0 & 4,0 & 5,0 \\
\hline $\mathbf{L}$ & $\lambda$ & & & & & & & & \\
\hline 5,654 & 0,05 & 283,8 & 181,9 & 115,8 & 77,9 & 55,1 & 41,7 & 26,7 & 19,0 \\
\hline 5,641 & 0,10 & 306,4 & 211,1 & 136,6 & 91,0 & 63,4 & 46,3 & 27,4 & 18,3 \\
\hline 5,430 & 0,15 & 314,9 & 223,4 & 149,1 & 100,9 & 69,6 & 50,1 & 28,8 & 18,4 \\
\hline 5,203 & 0,20 & 317,7 & 231,6 & 157,8 & 107,2 & 75,0 & 53,7 & 30,2 & 18,9 \\
\hline 4,981 & 0,25 & 319,1 & 237,4 & 164,3 & 112,9 & 78,3 & 56,1 & 31,6 & 19,4 \\
\hline 4,781 & 0,30 & 328,6 & 246,1 & 170,5 & 118,3 & 82,5 & 59,6 & 32,9 & 19,9 \\
\hline 4,570 & 0,35 & 320,8 & 243,2 & 171,2 & 119,1 & 84,1 & 60,2 & 33,4 & 20,2 \\
\hline 4,394 & 0,40 & 327,0 & 248,4 & 175,9 & 122,4 & 86,5 & 62,1 & 34,5 & 20,5 \\
\hline 4,074 & 0,50 & 330,7 & 255,3 & 183,5 & 127,8 & 90,7 & 65,3 & 36,0 & 21,2 \\
\hline
\end{tabular}

Tabela 8. NMA para o gráfico de EWMA $-\psi=0,9 ; \mathrm{n}=4 ; \phi=0,4$.

\begin{tabular}{|c|c|c|c|c|c|c|c|c|c|}
\hline \multicolumn{2}{|c|}{$\delta=(\xi-\xi 0) / \sigma_{\mathrm{e}}$} & 0,5 & 1,0 & 1,5 & 2,0 & 2,5 & 3,0 & 4,0 & 5,0 \\
\hline $\mathbf{L}$ & $\lambda$ & & & & & & & & \\
\hline 6,321 & 0,05 & 128,3 & 48,4 & 26,9 & 18,2 & 13,7 & 10,9 & 7,8 & 6,2 \\
\hline 6,851 & 0,10 & 157,9 & 57,6 & 29,1 & 18,1 & 12,9 & 10,0 & 6,8 & 5,2 \\
\hline 7,096 & 0,15 & 180,0 & 68,4 & 32,9 & 19,4 & 13,2 & 9,8 & 6,5 & 4,8 \\
\hline 7,240 & 0,20 & 200,4 & 80,0 & 37,9 & 21,5 & 14,0 & 10,1 & 6,3 & 4,6 \\
\hline 7,329 & 0,25 & 216,6 & 90,7 & 43,2 & 23,9 & 15,2 & 10,6 & 6,4 & 4,5 \\
\hline 7,392 & 0,30 & 229,4 & 101,2 & 48,6 & 26,9 & 16,6 & 11,4 & 6,6 & 4,5 \\
\hline 7,429 & 0,35 & 239,4 & 111,8 & 54,5 & 29,8 & 18,2 & 12,2 & 6,8 & 4,6 \\
\hline 7,454 & 0,40 & 250,6 & 122,0 & 60,8 & 33,3 & 20,2 & 13,3 & 7,1 & 4,6 \\
\hline 7,501 & 0,50 & 273,2 & 144,0 & 74,8 & 41,5 & 24,9 & 16,1 & 8,1 & 5,0 \\
\hline
\end{tabular}

Tabela 9. NMA para o gráfico de EWMA $-\psi=0,9 ; \mathrm{n}=4 ; \phi=0,8$.

\begin{tabular}{|c|c|c|c|c|c|c|c|c|c|}
\hline \multicolumn{2}{|c|}{$\delta=(\xi-\xi 0) / \sigma_{\mathrm{e}}$} & 0,5 & 1,0 & 1,5 & 2,0 & 2,5 & 3,0 & 4,0 & 5,0 \\
\hline $\mathbf{L}$ & $\lambda$ & & & & & & & & \\
\hline 8,90 & 0,05 & 195,3 & 85,9 & 47,7 & 31,0 & 22,6 & 17,5 & 12,1 & 9,2 \\
\hline 9,41 & 0,10 & 225,3 & 105,0 & 55,6 & 34,0 & 23,2 & 17,2 & 11,0 & 8,0 \\
\hline 9,56 & 0,15 & 246,3 & 122,7 & 65,2 & 38,5 & 25,4 & 18,1 & 11,0 & 7,7 \\
\hline 9,55 & 0,20 & 257,1 & 135,8 & 72,6 & 43,0 & 27,7 & 19,4 & 11,2 & 7,5 \\
\hline 9,50 & 0,25 & 272,0 & 149,2 & 81,7 & 48,3 & 30,7 & 21,0 & 11,7 & 7,6 \\
\hline 9,40 & 0,30 & 277,8 & 159,6 & 89,0 & 52,8 & 33,5 & 22,6 & 12,2 & 7,7 \\
\hline 9,30 & 0,35 & 289,3 & 170,8 & 96,9 & 58,0 & 36,8 & 24,6 & 12,9 & 8,0 \\
\hline 9,17 & 0,40 & 293,4 & 179,0 & 103,7 & 62,2 & 39,4 & 26,4 & 13,7 & 8,3 \\
\hline 8,88 & 0,50 & 298,4 & 190,8 & 114,6 & 70,1 & 44,8 & 29,8 & 15,1 & 8,9 \\
\hline
\end{tabular}


caso esteja em uso o gráfico de $\bar{X}$, e NMA $=195,30$ (Tabela 9), caso esteja em uso o gráfico de EWMA $(\lambda=0,05)$. Quando há um grande deslocamento $(\delta=5,0)$, tem-se para o gráfico de $\bar{X}$ um NMA $=22,6$ (Tabela 4), enquanto que, para o gráfico de EWMA $(\lambda=0,05)$, o NMA $=9,20$ (Tabela 9).

\section{Efeito da autocorrelação no planejamento dos gráficos de controle}

O planejamento de um gráfico de controle consiste na determinação de seus parâmetros de construção: $n, h$ e $L$. Os gráficos de controle são dispositivos estatísticos que sinalizam alterações no processo; eles podem ser vistos como uma sequência de testes de hipóteses, isto é, após a retirada de cada amostra, julgamos com base em informações extraídas dela, se o processo está sob controle ou fora de controle. Como em todo teste de hipóteses, tem-se aqui também os erros do tipo I e II. O erro do tipo I ocorre quanto erroneamente julgamos que o processo está fora de controle. Na linguagem do CEP - Controle Estatístico de Processos, o erro do tipo I é chamado de alarme falso. O alarme falso deve ser evitado ao máximo, pois ele gera intervenções desnecessárias no processo, na busca de causas especiais inexistentes. O usuário dos gráficos de controle deve definir a incidência máxima suportável de alarmes falsos ou, em outras palavras, o mínimo TMAF. O erro do tipo II, ou ainda risco $\beta$, ocorre quanto erroneamente julgamos que o processo está sob controle. Na linguagem do CEP, a probabilidade da não ocorrência do erro do tipo II é chamada de poder de detecção da carta de controle. No caso em que a média do processo não oscila, o NMA nada mais é que o inverso do poder de detecção. Trabalhar com grandes amostras pode prejudicar o desempenho do gráfico, pois amostras grandes implicam em longos intervalos entre retirada de amostras, por conseguinte, mudanças na média podem passar despercebidas por um bom tempo. A medida de desempenho TES é mais adequada em relação ao NMA porque leva ao melhor esquema de monitoramento, considerando o tamanho das amostras e o intervalo de tempo entre retirada de amostras. Os valores ótimos de $n, h$ e $L$ são aqueles que minimizam o TES sob a restrição do mínimo TMAF permitido e da máxima taxa de inspeção TI $=n / h$ disponível. Epprecht e Santos (1998) exploram este método para projetar gráficos quando não há autocorrelação no processo. A relação entre o TES e o NMA é simples: TES $/ h=\mathrm{NMA}-0,5$ (para detalhes consultar COSTA; EPPRECHT; CARPINETTI, 2005). Nas Tabelas 10 a 18, aparecem em destaque (negrito) os valores mínimos do TES.

Nas Tabelas 10 e 11, estão os valores ótimos de $n, h$ e $L$ do gráfico de $\bar{X}$ e de EWMA, para o caso em que as observações de $X$ são independentes. O TMAF é de 370,4 horas e a TI de 8 unidades por hora. No gráfico de $\bar{X}$ (ver Tabela 10), para um pequeno deslocamento da média $\left(\delta \sigma_{e}=0,5\right)$, a melhor opção é tomar amostras de tamanho 20 em intervalos de 2,5 horas; já para deslocamentos maiores $\left(\delta \sigma_{e}=2,0\right)$, a melhor opção é tomar amostras de tamanho 4 a cada meia hora. No esquema EWMA (ver Tabela 11), amostras unitárias a cada 7,5 minutos são preferíveis quando se utiliza valores de $\lambda$ próximos a zero $(\lambda=0,05)$, para pequenos e grandes deslocamentos. Para valores crescentes de $\lambda$, amostras maiores se tornam melhores na sinalização de pequenos deslocamentos.

\subsection{O efeito da autocorrelação no planejamento do gráfico de $\bar{X}$}

Na presença da autocorrelação, se a parcela da variabilidade de $X$ resultante do movimento oscilatório da média do processo, em relação a sua variabilidade total, é grande, não há vantagens em se trabalhar

Tabela 10. TES do gráfico de $\bar{X}$ (processo independente).

\begin{tabular}{|c|c|c|c|c|c|c|}
\hline \multicolumn{7}{|c|}{$\phi=\mathbf{0}$} \\
\hline $\mathrm{n}$ & 1 & 4 & 8 & 12 & 16 & 20 \\
\hline $\mathrm{h}$ & 0,125 & 0,500 & 1,000 & 1,500 & 2,000 & 2,500 \\
\hline $\mathrm{L}$ & 3,585 & 3,205 & 3,000 & 2,874 & 2,782 & 2,709 \\
\hline \multicolumn{4}{|c|}{$\delta$} & \multicolumn{3}{|c|}{ TES } \\
\hline 0,0 & 370,40 & 370,40 & 370,40 & 370,40 & 370,40 & 370,40 \\
\hline 0,5 & 120,03 & 36,16 & 17,23 & 11,09 & 8,23 & 6,61 \\
\hline 1,0 & 25,58 & 4,13 & 1,82 & 1,33 & 1,25 & 1,35 \\
\hline 1,5 & 6,68 & 0,94 & 0,62 & 0,77 & 1,00 & 1,25 \\
\hline 2,0 & 2,15 & 0,39 & 0,50 & 0,75 & 1,00 & 1,25 \\
\hline 3,0 & 0,38 & 0,25 & 0,50 & 0,75 & 1,00 & 1,25 \\
\hline 4,0 & 0,13 & 0,25 & 0,50 & 0,75 & 1,00 & 1,25 \\
\hline 5,0 & $\mathbf{0 , 0 7}$ & 0,25 & 0,50 & 0,75 & 1,00 & 1,25 \\
\hline
\end{tabular}


com amostras maiores que um, pois o menor TES é sempre obtido com observações individuais (ver Tabelas 12 e 13). Para valores grandes de $\psi$, os limites do gráfico de controle de $\bar{X}$ se alargam de $f(n, \psi)$, por conseguinte, a carta se torna muita lenta na sinalização; seu uso só se justifica quando o processo é altamente capaz de atender às especificações de $X$, mesmo com a sua média oscilando. Os gráficos de controle de CUSUM e EWMA são alternativas para um melhor poder de detecção, Lu e Reynolds Junior (1999a, 2001). Como trabalhos anteriores evidenciaram que as cartas de controle de EWMA e CUSUM têm desempenho similar (veja, por exemplo, VAN BRACKLE III; REYNOLDS JUNIOR, 1997), optou-se pela carta de EWMA por sua simplicidade diante do gráfico CUSUM.

\subsection{O efeito da autocorrelação no planejamento do gráfico de EWMA}

$\mathrm{Na}$ presença da autocorrelação, o fator de ponderação $(\lambda)$ do gráfico EWMA exerce forte influência no planejamento da carta de controle. As Tabelas 14 a 18 apresentam os TES quando está em uso o gráfico de controle EWMA para diversas combinações de $\lambda(\lambda=0,05 ; 0,25 ; 0,50 ; 0,80$ e 1,00$)$, grau de autocorrelação $\phi(\phi=0,4$ e 0,8$)$, proporção da variabilidade de $\mathrm{X}$ atribuída à oscilação da média $(\psi=0,5$ e 0,9$)$ e amostras de tamanho $n(n=1,2,4$ e 8$)$. Dependendo da magnitude do deslocamento da média, há vantagens em se trabalhar com amostras maiores que um, isto é, o menor valor do TES é observado com $\mathrm{n}>1$. Exemplo, para $\lambda=0,5, \phi=0,4, \psi=0,9 \mathrm{e}$ $\delta=1,5, \mathrm{n}=4$ (Tabela 16) leva ao menor valor de TES. Para observações independentes (Tabela 11), ou altamente correlacionadas $(\phi=0,8$ - Tabela 16), $\mathrm{n}=8$ leva ao menor valor de TES. Para valores de $\lambda$ próximos a 1, o gráfico EWMA possui comportamento similar ao do gráfico de $\bar{X}$, ou seja, se $\psi=0,9$, então amostras unitárias levam ao melhor desempenho (Tabela 18). Verifica-se ainda o quão rápido é o esquema EWMA na sinalização de pequenos e grandes desvios na média do processo em relação ao gráfico de $\bar{X}$ quando há forte oscilação da média $(\psi=0,9)$ e elevado grau de autocorrelação $(\phi=0,8)$. Exemplo,

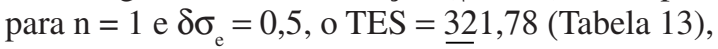
caso esteja em uso o gráfico de $\bar{X}$, e TES $=235,05$ (Tabela $14-\lambda=0,05$ ), caso esteja em uso o gráfico

Tabela 11. TES do gráfico EWMA (processo independente); $\psi=0$.

\begin{tabular}{cccccccccccccc}
\hline \multicolumn{1}{c}{$\lambda=\mathbf{0 , 0 5}$} & \multicolumn{1}{c}{$\lambda=\mathbf{0 , 2 5}$} \\
\hline $\mathbf{n}$ & $\mathbf{1}$ & $\mathbf{2}$ & $\mathbf{4}$ & $\mathbf{8}$ & $\mathbf{1}$ & $\mathbf{2}$ & $\mathbf{4}$ & $\mathbf{8}$ & $\mathbf{1}$ & $\mathbf{2}$ & $\mathbf{4}$ & $\mathbf{8}$ \\
$\mathbf{h}$ & $\mathbf{0 , 1 2 5}$ & $\mathbf{0 , 2 5}$ & $\mathbf{0 , 5 0}$ & $\mathbf{1 , 0 0}$ & $\mathbf{0 , 1 2 5}$ & $\mathbf{0 , 2 5}$ & $\mathbf{0 , 5 0}$ & $\mathbf{1 , 0 0}$ & $\mathbf{0 , 1 2 5}$ & $\mathbf{0 , 2 5}$ & $\mathbf{0 , 5 0}$ & $\mathbf{1 , 0 0}$ \\
$\mathbf{L}$ & $\mathbf{3 , 2 5 7}$ & $\mathbf{3 , 0 2 2}$ & $\mathbf{3 , 0 0 0}$ & $\mathbf{3 , 5 2 0}$ & $\mathbf{3 , 5 3 2}$ & $\mathbf{3 , 3 3 1}$ & $\mathbf{3 , 1 2 2}$ & $\mathbf{4 , 0 9 1}$ & $\mathbf{3 , 5 7 5}$ & $\mathbf{3 , 3 8 8}$ & $\mathbf{3 , 1 9 2}$ & $\mathbf{4 , 2 0 9}$ \\
\hline$\delta$ & & & \multicolumn{1}{c}{$\mathbf{T E S}$} \\
\hline $\mathbf{0 , 0}$ & 370,40 & 370,40 & 370,40 & 370,40 & 370,40 & 370,40 & 370,40 & 370,40 & 370,40 & 370,40 & 370,40 & 370,40 \\
$\mathbf{0 , 5}$ & 5,42 & $\mathbf{5 , 2 5}$ & 6,40 & 6,16 & 16,42 & 8,68 & 5,84 & $\mathbf{4 , 5 6}$ & 41,35 & 20,08 & 10,17 & $\mathbf{5 , 3 9}$ \\
$\mathbf{1 , 0}$ & $\mathbf{1 , 7 9}$ & 2,07 & 2,73 & 2,82 & 2,20 & 1,67 & $\mathbf{1 , 6 2}$ & 1,65 & 5,09 & 2,49 & 1,69 & $\mathbf{1 , 3 4}$ \\
$\mathbf{1 , 5}$ & $\mathbf{1 , 0 6}$ & 1,28 & 1,72 & 1,82 & 0,85 & $\mathbf{0 , 8 2}$ & 0,91 & 0,97 & 1,32 & 0,85 & 0,75 & $\mathbf{0 , 6 8}$ \\
$\mathbf{2 , 0}$ & $\mathbf{0 , 7 5}$ & 0,93 & 1,26 & 1,35 & $\mathbf{0 , 5 0}$ & 0,54 & 0,63 & 0,64 & 0,57 & 0,47 & $\mathbf{0 , 4 6}$ & 0,51 \\
$\mathbf{2 , 5}$ & $\mathbf{0 , 5 8}$ & 0,72 & 0,99 & 1,07 & $\mathbf{0 , 3 5}$ & 0,39 & 0,47 & 0,52 & 0,34 & $\mathbf{0 , 3 1}$ & $\mathbf{0 , 3 1}$ & 0,50 \\
$\mathbf{3 , 0}$ & $\mathbf{0 , 4 7}$ & 0,59 & 0,82 & 0,87 & $\mathbf{0 , 2 7}$ & 0,31 & 0,35 & 0,50 & $\mathbf{0 , 2 3}$ & $\mathbf{0 , 2 3}$ & 0,26 & 0,50 \\
$\mathbf{4 , 0}$ & $\mathbf{0 , 3 4}$ & 0,44 & 0,61 & 0,59 & $\mathbf{0 , 1 8}$ & 0,21 & 0,26 & 0,50 & $\mathbf{0 , 1 4}$ & $\mathbf{0 , 1 4}$ & 0,25 & 0,50 \\
$\mathbf{5 , 0}$ & $\mathbf{0 , 2 7}$ & 0,35 & 0,48 & 0,51 & $\mathbf{0 , 1 4}$ & 0,15 & 0,25 & 0,50 & $\mathbf{0 , 0 9}$ & 0,13 & 0,25 & 0,50 \\
\hline
\end{tabular}

Tabela 12. TES do gráfico de $\bar{X}(\phi=+0,4)$.

\begin{tabular}{|c|c|c|c|c|c|c|c|c|c|c|c|c|c|c|c|}
\hline & \multicolumn{5}{|c|}{$\psi=\mathbf{0 , 1}$} & \multicolumn{5}{|c|}{$\psi=\mathbf{0 , 5}$} & \multicolumn{5}{|c|}{$\psi=\mathbf{0 , 9}$} \\
\hline $\mathrm{n}$ & 1 & 4 & 8 & 12 & 16 & 1 & 4 & 8 & 12 & 16 & 1 & 4 & 8 & 12 & 16 \\
\hline h & 0,125 & 0,5 & 1 & 1,5 & 2 & 0,125 & 0,5 & 1 & 1,5 & 2 & 0,125 & 0,5 & 1 & 1,5 & 2 \\
\hline $\mathbf{L}$ & $3, \mathbf{5 8 5}$ & 3,205 & 2,998 & 2,871 & 2,777 & 3,584 & 3,201 & 2,992 & 2,864 & 2,769 & 3,582 & 3,198 & 2,990 & 2,861 & 2,767 \\
\hline$\delta$ & & & & & & & & TES & & & & & & & \\
\hline 0,0 & 370,40 & 370,40 & 370,40 & 370,40 & 370,40 & 370,40 & 370,40 & 370,40 & 370,40 & 370,40 & 370,40 & 370,40 & 370,40 & 370,40 & 370,40 \\
\hline 0,5 & 130,38 & 56,30 & 40,50 & 35,90 & 34,19 & 190,72 & 164,42 & 166,60 & 171,04 & 175,25 & 315,91 & 321,06 & 325,34 & 328,02 & 329,89 \\
\hline 1,0 & 29,80 & 7,88 & 5,28 & 4,76 & 4,70 & 62,73 & 47,70 & 49,82 & 53,01 & 56,02 & 214,09 & 225,46 & 235,53 & 242,14 & 247,03 \\
\hline 1,5 & 8,15 & 1,85 & 1,37 & 1,39 & 1,52 & 21,98 & 15,84 & 17,24 & 19,01 & 20,68 & 132,34 & 144,67 & 156,12 & 163,92 & 169,86 \\
\hline 2,0 & 2,70 & 0,66 & 0,65 & 0,83 & 1,04 & 8,63 & 6,17 & 7,05 & 8,06 & 9,01 & 80,48 & 91,10 & 101,33 & 108,51 & 114,09 \\
\hline 3,0 & 0,49 & 0,27 & 0,50 & 0,75 & 1,00 & 1,85 & 1,43 & 1,82 & 2,23 & 2,63 & 31,12 & 37,53 & 44,12 & 48,95 & 52,83 \\
\hline 4,0 & 0,15 & 0,25 & 0,50 & 0,75 & 1,00 & 0,57 & 0,53 & 0,78 & 1,05 & 1,31 & 13,18 & 16,84 & 20,83 & 23,87 & 26,38 \\
\hline 5,0 & 0,08 & 0,25 & 0,50 & 0,75 & 1,00 & 0,22 & 0,31 & 0,55 & 0,79 & 1,04 & 6,13 & 8,24 & 10,70 & 12,63 & 14,26 \\
\hline
\end{tabular}


Tabela 13. TES do gráfico de $\bar{X}(\phi=+0,8)$.

\begin{tabular}{|c|c|c|c|c|c|c|c|c|c|c|c|c|c|c|c|}
\hline & \multicolumn{5}{|c|}{$\psi=\mathbf{0 , 1}$} & \multicolumn{5}{|c|}{$\psi=\mathbf{0 , 5}$} & \multicolumn{5}{|c|}{$\psi=\mathbf{0 , 9}$} \\
\hline n & 1 & 4 & 8 & 12 & 16 & 1 & 4 & 8 & 12 & 16 & 1 & 4 & 8 & 12 & 16 \\
\hline h & 0,125 & 0,5 & 1 & 1,5 & 2 & 0,125 & $\mathbf{0 , 5}$ & 1 & 1,5 & 2 & 0,125 & 0,5 & 1 & 1,5 & 2 \\
\hline $\mathbf{L}$ & 3,585 & 3,201 & 2,982 & $\mathbf{2 , 8 3 9}$ & 2,730 & 3,578 & 3,153 & 2,907 & 2,754 & 2,643 & 3,536 & 3,105 & 2,870 & 2,868 & 2,619 \\
\hline$\delta$ & & & & & & & & TES & & & & & & & \\
\hline 0,0 & 370,40 & 370,40 & 370,40 & 370,40 & 370,40 & 370,40 & 370,40 & 370,40 & 370,40 & 370,40 & 370,40 & 370,40 & 370,40 & 370,40 & 370,40 \\
\hline 0,5 & 130,88 & 57,19 & 41,07 & 36,20 & 34,34 & 195,23 & 168,00 & 168,06 & 171,68 & 175,55 & 321,78 & 322,85 & 325,89 & 328,24 & 329,99 \\
\hline 1,0 & 30,20 & 8,29 & 5,48 & 4,86 & 4,74 & 66,98 & 50,61 & 51,06 & 53,58 & 56,29 & 227,00 & 229,66 & 236,87 & 242,70 & 247,28 \\
\hline 1,5 & 8,42 & 2,00 & 1,42 & 1,40 & 1,53 & 24,82 & 17,64 & 18,04 & 19,38 & 20,86 & 146,70 & 149,68 & 157,80 & 164,63 & 170,19 \\
\hline 2,0 & 2,86 & 0,70 & 0,66 & 0,83 & 1,04 & 10,42 & 7,23 & 7,53 & 8,29 & 9,11 & 93,28 & 95,87 & 103,01 & 109,23 & 114,43 \\
\hline 3,0 & 0,53 & 0,27 & 0,50 & 0,75 & 1,00 & 2,51 & 1,75 & 1,96 & 2,29 & 2,66 & 39,48 & 41,05 & 45,47 & 49,55 & 53,11 \\
\hline 4,0 & 0,16 & 0,25 & 0,50 & 0,75 & 1,00 & 0,78 & 0,60 & 0,81 & 1,06 & 1,32 & 18,33 & 19,25 & 21,82 & 24,32 & 26,59 \\
\hline 5,0 & $\mathbf{0 , 0 8}$ & 0,25 & 0,50 & 0,75 & 1,00 & 0,28 & 0,32 & 0,55 & 0,79 & 1,04 & $\mathbf{9 , 3 0}$ & 9,86 & 11,40 & 12,95 & 14,41 \\
\hline
\end{tabular}

Tabela 14. TES do gráfico EWMA; $\lambda=0,05$.

\begin{tabular}{|c|c|c|c|c|c|c|c|c|c|c|c|c|c|c|c|c|}
\hline & \multicolumn{4}{|c|}{$\phi=+\mathbf{0 , 4} ; \psi=\mathbf{0 , 5}$} & \multicolumn{4}{|c|}{$\phi=+\mathbf{0 , 8} ; \psi=\mathbf{0 , 5}$} & \multicolumn{4}{|c|}{$\phi=+\mathbf{0 , 4} ; \psi=\mathbf{0 , 9}$} & \multicolumn{4}{|c|}{$\phi=+\mathbf{0 , 8} ; \psi=\mathbf{0 , 9}$} \\
\hline n & 1 & 2 & 4 & 8 & 1 & 2 & 4 & 8 & 1 & 2 & 4 & 8 & 1 & 2 & 4 & 8 \\
\hline h & 0,125 & 0,25 & $\mathbf{0 , 5 0}$ & 1,00 & 0,125 & 0,25 & $\mathbf{0 , 5 0}$ & 1,00 & 0,125 & 0,25 & $\mathbf{0 , 5 0}$ & 1,00 & 0,125 & 0,25 & $\mathbf{0 , 5 0}$ & 1,00 \\
\hline $\mathbf{L}$ & 4,066 & 4,933 & 6,815 & $\mathbf{1 0 , 8 1 9}$ & 6,240 & 7,504 & 9,351 & 12,436 & 4,602 & 5,184 & 7,038 & 11,156 & 7,781 & 8,495 & 9,970 & 12,878 \\
\hline$\delta$ & \multicolumn{16}{|c|}{ TES } \\
\hline 0,0 & 370,40 & 370,40 & 370,40 & 370,4 & 370,40 & 370,4 & 370,40 & 370,40 & 370,40 & 3 & $3 / 0$ & & & & & \\
\hline 0,5 & 18,92 & 15,61 & 17,88 & 26,07 & 56,98 & 40,28 & 32,44 & 32,81 & 118,39 & 96,14 & 98,02 & 123,32 & 235,05 & 199,13 & 165,20 & 148,64 \\
\hline 1,0 & 4,52 & 4,89 & 6,51 & 10,33 & 12,62 & 10,56 & 10,57 & 12,54 & 32,55 & 27,28 & 31,37 & 45,96 & 107,25 & 78,83 & 61,68 & 58,71 \\
\hline 1,5 & 2,32 & 2,79 & 3,92 & 6,3 & 5,37 & 5,3 & 5,98 & & 13,26 & 12,45 & 16 , & & 51,48 & 37,13 & 31,10 & 31,83 \\
\hline 2,0 & 1,54 & 1,94 & 2,78 & 4,55 & 3,15 & 3,45 & 4,13 & 5,36 & 7,23 & 7,56 & 10,42 & 16,97 & 28,01 & 21,14 & 19,01 & 21,14 \\
\hline 2,5 & 1,15 & 1,48 & 2,15 & 3,54 & 2,17 & 2,54 & 3.13 & 4,14 & 4,70 & 5,34 & 7,63 & 12,67 & 16,84 & 13,76 & 13,34 & 15,59 \\
\hline 3,0 & 0,91 & 1,20 & 1,76 & 2,90 & 1,64 & 2,00 & 2,53 & 3,38 & 3,41 & 4,09 & 5,99 & 10,08 & 11,25 & 9,77 & 10,09 & 12,28 \\
\hline 4,0 & 0,65 & 0,87 & 1,28 & 2,1 & 1,10 & 1,41 & 1,8 & 2,47 & 2,17 & 2,77 & 4,17 & 7,10 & 6,10 & 5,97 & 6,71 & 8,53 \\
\hline 5,0 & 0,59 & 0,68 & 1,01 & 1,68 & 0,83 & 1,08 & 1,42 & 1,94 & 1,58 & 2,08 & 3,19 & 5,47 & 3,94 & 4,21 & 4,97 & 6,53 \\
\hline
\end{tabular}

Tabela 15. TES do gráfico EWMA; $\lambda=0,25$.

\begin{tabular}{|c|c|c|c|c|c|c|c|c|c|c|c|c|c|c|c|c|}
\hline & \multicolumn{4}{|c|}{$\phi=+\mathbf{0 , 4} ; \psi=\mathbf{0 , 5}$} & \multicolumn{4}{|c|}{$\phi=+\mathbf{0 , 8} ; \psi=\mathbf{0 , 5}$} & \multicolumn{4}{|c|}{$\phi=+\mathbf{0 , 4} ; \psi=\mathbf{0 , 9}$} & \multicolumn{4}{|c|}{$\phi=+\mathbf{0 , 8} ; \psi=\mathbf{0 , 9}$} \\
\hline $\mathbf{n}$ & 1 & 2 & 4 & 8 & 1 & 2 & 4 & 8 & 1 & 2 & 4 & 8 & 1 & 2 & 4 & 8 \\
\hline $\mathbf{h}$ & 0,125 & 0,25 & $\mathbf{0 , 5 0}$ & 1,00 & 0,125 & 0,25 & $\mathbf{0 , 5 0}$ & 1,00 & 0,125 & 0,25 & $\mathbf{0 , 5 0}$ & 1,00 & 0,125 & 0,25 & $\mathbf{0 , 5 0}$ & 1,00 \\
\hline $\mathbf{L}$ & 4,173 & 5,319 & 7,635 & 12,600 & 5,350 & 7,127 & 9,725 & 14,040 & 4,609 & 5,560 & 7,905 & 13,005 & 6,355 & 7,883 & 10,307 & 14,550 \\
\hline$\delta$ & \multicolumn{16}{|c|}{ TES } \\
\hline 0,0 & 370,40 & 370,40 & 370,40 & 370,40 & 370,40 & 370,40 & 370,40 & 370,40 & 370,40 & 370,40 & 370,40 & 370,40 & 370,40 & 370,40 & 370,40 & 370,40 \\
\hline 0,5 & 64,23 & 43,23 & 36,11 & 42,25 & 120,08 & 94,24 & 68,95 & 55,23 & 231,92 & 206,30 & 195,76 & 211,96 & 299,18 & 283,12 & 254,79 & 237,66 \\
\hline 1,0 & 10,96 & 7,38 & 7,14 & 10,04 & 29,32 & 20,76 & 14,96 & 13,32 & 99,77 & 78,20 & 73,23 & 86,70 & 186,71 & 159,52 & 128,87 & 109,44 \\
\hline 1,5 & 3,35 & 2,69 & 3,10 & 4,77 & 9,75 & 7,17 & $\mathbf{5 , 8 0}$ & 6,00 & 43,56 & 32,42 & 31,43 & 40,49 & 107,49 & 85,92 & 64,84 & 53,81 \\
\hline 2,0 & 1,54 & 1,47 & 1,88 & 3,02 & 4,16 & 3,38 & 3,14 & 3,65 & 21,32 & 15,66 & 15,99 & 22,29 & 62,75 & 48,44 & 35,60 & 30,23 \\
\hline 2,5 & 0,91 & 0,98 & 1,33 & 2,18 & 2,17 & 1,96 & 2,05 & 2,58 & 11,25 & $\mathbf{8 , 5 8}$ & 9,47 & 13,95 & 38,23 & 28,89 & 21,43 & 18,87 \\
\hline 3,0 & 0,63 & 0,73 & 1,02 & 1,70 & 1,31 & 1,32 & 1,50 & 1,98 & 6,61 & 5,32 & 6,26 & 9,64 & 24,35 & 18,26 & 13,80 & 12,89 \\
\hline 4,0 & 0,38 & 0,48 & 0,70 & 1,18 & 0,64 & 0,76 & 0,97 & 1,35 & 2,86 & 2,65 & 3,46 & 5,65 & 11,05 & 8,52 & 7,00 & 7,28 \\
\hline 5,0 & $\mathbf{0 , 2 7}$ & 0,36 & 0,53 & 0,87 & 0,41 & 0,53 & 0,72 & 1,02 & 1,58 & 1,64 & 2,30 & 3,88 & 5,76 & 4,69 & 4,27 & 4,84 \\
\hline
\end{tabular}

Tabela 16. TES do gráfico EWMA; $\lambda=0,50$.

\begin{tabular}{|c|c|c|c|c|c|c|c|c|c|c|c|c|c|c|c|c|}
\hline & \multicolumn{4}{|c|}{$\phi=+\mathbf{0 , 4} ; \psi=\mathbf{0 , 5}$} & \multicolumn{4}{|c|}{$\phi=+\mathbf{0 , 8} ; \psi=\mathbf{0 , 5}$} & \multicolumn{4}{|c|}{$\phi=+\mathbf{0 , 4} ; \psi=\mathbf{0 , 9}$} & \multicolumn{4}{|c|}{$\phi=+\mathbf{0 , 8} ; \psi=\mathbf{0 , 9}$} \\
\hline n & 1 & 2 & 4 & 8 & 1 & 2 & 4 & 8 & 1 & 2 & 4 & 8 & 1 & 2 & 4 & 8 \\
\hline h & 0,125 & 0,25 & $\mathbf{0 , 5 0}$ & 1,00 & 0,125 & 0,25 & $\mathbf{0 , 5 0}$ & 1,00 & 0,125 & 0,25 & $\mathbf{0 , 5 0}$ & 1,00 & 0,125 & 0,25 & $\mathbf{0 , 5 0}$ & 1,00 \\
\hline $\mathbf{L}$ & 3,982 & 5,280 & 7,771 & 12,951 & $\mathbf{4 , 5 2 7}$ & 6,280 & 9,100 & 13,918 & 4,269 & 5,450 & 8,031 & 13,355 & 5,073 & 6,762 & $\mathbf{9 , 5 7 0}$ & 14,415 \\
\hline$\delta$ & \multicolumn{16}{|c|}{ TES } \\
\hline 0,0 & 370,40 & 370,40 & 370,40 & 370,40 & 370,40 & 370,40 & 370,40 & 370,40 & 370,40 & 370,40 & 370,40 & 370, & 370,40 & 370,4 & 370,40 & 370,40 \\
\hline 0,5 & 111,10 & 85,84 & 70,91 & 74,44 & 149,07 & 128,42 & 105,16 & 88,6 & 278, & 261,09 & 258,12 & 267 , & 309, & & 291,73 & 281,50 \\
\hline 1,0 & 23,81 & 15,87 & 13,07 & 15,54 & 41,73 & 32,06 & 24,23 & 20,08 & 150,65 & 130,59 & 127,39 & 139,68 & 205,74 & 92,44 & 174,57 & 159,58 \\
\hline 1,5 & 6,88 & 4,62 & 4,28 & 5,72 & 14,02 & 10,67 & 8,15 & 7,37 & 77,17 & 63,30 & 61,60 & 70,99 & 126,18 & 114,00 & 98,08 & 86,84 \\
\hline 2,0 & 2,62 & 1,96 & 2,06 & 3,02 & 5,78 & 4,50 & 3,68 & 3,74 & 40,72 & 32,39 & 32,13 & 39,10 & 77,80 & 68,30 & 56,61 & 49,11 \\
\hline 2,5 & 1,27 & 1,07 & 1,26 & 1,95 & 2,78 & 2,26 & 2,05 & 2,32 & 22,60 & 17,75 & 18,00 & 23,15 & 48,61 & 41,81 & 34,38 & 29,98 \\
\hline 3,0 & $\mathbf{0 , 7 3}$ & 0,70 & 0,88 & 1,40 & 1,51 & 1,33 & 1,32 & 1,62 & 13,33 & 10,38 & 10,92 & 14,71 & 31,63 & 26,77 & 21,83 & 19,46 \\
\hline 4,0 & 0,34 & 0,39 & 0,53 & 0,86 & 0,58 & 0,60 & 0,71 & 0,98 & 5,24 & 4,33 & 4,90 & 7,21 & 14,41 & 12,20 & 10,12 & 9,49 \\
\hline 5,0 & 0,21 & 0,26 & 0,36 & 0,60 & 0,30 & 0,36 & 0,48 & 0,67 & 2,51 & 2,21 & 2,74 & 4,29 & 7,32 & 6,34 & 5,44 & 5,48 \\
\hline
\end{tabular}


Tabela 17. TES do gráfico EWMA; $\lambda=0,80$.

\begin{tabular}{|c|c|c|c|c|c|c|c|c|c|c|c|c|c|c|c|c|}
\hline & \multicolumn{4}{|c|}{$\phi=+\mathbf{0 , 4} ; \psi=\mathbf{0 , 5}$} & \multicolumn{4}{|c|}{$\phi=+\mathbf{0 , 8} ; \psi=\mathbf{0 , 5}$} & \multicolumn{4}{|c|}{$\phi=+\mathbf{0 , 4} ; \psi=\mathbf{0 , 9}$} & \multicolumn{4}{|c|}{$\phi=+\mathbf{0 , 8} ; \psi=\mathbf{0 , 9}$} \\
\hline $\mathbf{n}$ & 1 & 2 & 4 & 8 & 1 & 2 & 4 & 8 & 1 & 2 & 4 & 8 & 1 & 2 & 4 & 8 \\
\hline h & 0,125 & 0,25 & 0,50 & 1,00 & 0,125 & 0,25 & 0,50 & 1,00 & 0,125 & 0,25 & 0,50 & 1,00 & 0,125 & 0,25 & 0,50 & 1,00 \\
\hline $\mathbf{L}$ & & 5,112 & 7,751 & 13.040 & 3,890 & 5,450 & 8,247 & 13,421 & 3,848 & 5,234 & 8,003 & 13,421 & & 5,664 & 8,581 & \\
\hline$\delta$ & \multicolumn{16}{|c|}{ TES } \\
\hline 0,0 & 370,40 & 370,40 & & & & & & 270 & 370,40 & & & & & & & 370 \\
\hline 0,5 & 159,94 & 131,88 & 120,00 & 2,83 & & 155 & & & & & & & & &, 63 & 310,12 \\
\hline 1,0 & 44,43 & 31,92 & 27,36 & 29,60 & 55,00 & 43 & 36,41 & 33, & 191,69 & 183,68 & 5,29 & 196 & 218,07 & 212,24 & 207,52 & 206,07 \\
\hline 1,5 & 14,19 & 9,63 & & & 19,38 & 14,99 & 12,13 & 11,52 & 110 , & 103,60 & 105,93 & 117,12 & 138,56 & 133,84 & 128,37 & 127,3 \\
\hline 2,0 & 5,34 & 3,61 & 3,31 & 4,19 & 8,05 & 6,14 & 5,02 & & 64,23 & 59,36 & 61,71 & 70,21 & 86,86 & 83,07 & 78,95 & 78,17 \\
\hline 2,5 & 2,33 & 1,66 & 1,64 & 2,24 & 3,82 & 2,91 & 2,48 & 2,61 & 37,59 & 34,82 & 36,92 & 43.50 & 55,25 & 52,61 & 49,72 & 49,03 \\
\hline 3,0 & 1,19 & 0,90 & 0,96 & 1,38 & 1,97 & 1,55 & 1,38 & 1,58 & 22,98 & 21,29 & 22,77 & 27,86 & 36,32 & 34,00 & 32,23 & 32,10 \\
\hline 4,0 & 0,42 & 0,37 & 0,45 & 0,72 & 0,65 & 0,55 & 0,57 & 0,78 & 9,32 & 8,70 & 9,76 & 12,77 & 16,76 & 15,76 & 14,87 & 15,06 \\
\hline 5,0 & 0,20 & 0,21 & 0,30 & 0,54 & 0,27 & 0,26 & 0,33 & 0,55 & 4,29 & 4,10 & 4,80 & 6,67 & 8,51 & 8,05 & 7,66 & 8,02 \\
\hline
\end{tabular}

Tabela 18. TES do gráfico EWMA; $\lambda=1,0$.

\begin{tabular}{|c|c|c|c|c|c|c|c|c|c|c|c|c|c|c|c|c|}
\hline & \multicolumn{4}{|c|}{$\phi=+\mathbf{0 , 4} ; \psi=\mathbf{0 , 5}$} & \multicolumn{4}{|c|}{$\phi=+0,8 ; \psi=0,5$} & \multicolumn{4}{|c|}{$\phi=+\mathbf{0 , 4} ; \psi=\mathbf{0 , 9}$} & \multicolumn{4}{|c|}{$\phi=+\mathbf{0 , 8} ; \psi=\mathbf{0 , 9}$} \\
\hline $\mathbf{n}$ & 1 & 2 & 4 & 8 & 1 & 2 & 4 & 8 & 1 & 2 & 4 & 8 & 1 & 2 & 4 & 8 \\
\hline h & 0,125 & 0,25 & $\mathbf{0 , 5 0}$ & 1,00 & 0,125 & 0,25 & $\mathbf{0 , 5 0}$ & 1,00 & 0,125 & 0,25 & $\mathbf{0 , 5 0}$ & & 0,125 & 0,25 & $\mathbf{0 , 5 0}$ & \\
\hline $\mathbf{L}$ & & 5,005 & 7,714 & 13,041 & 3,575 & 4,992 & 7,710 & 13,030 & 3,582 & 5,082 & 7,972 & 13,441 & 3,534 & 5,044 & 7,941 & 13,440 \\
\hline$\delta$ & \multicolumn{16}{|c|}{ TES } \\
\hline 0,0 & 70,40 & & & & & & & & & & & & & & & \\
\hline 0,5 & 192,95 & 166,80 & 156,28 & 159,64 & & & & & & & & & & & & \\
\hline 1,0 & 63,91 & 49,02 & 43,09 & 46,25 & 66,43 & 53,18 & 46,94 & 47,14 & 212,46 & 217,00 & 224,43 & 233,21 & 224,28 & 224,72 & 225,00 & 234,4 \\
\hline 1,5 & 22,39 & 16,09 & 14,09 & 15,5 & 24,49 & 18,83 & 16,01 & 16 , & 132,00 & 134,93 & 142,87 & & 144,82 & 144,87 & 146,25 & 154 \\
\hline 2,0 & 8,78 & 6,11 & 5,38 & 6,2 & 10,40 & 7,67 & & & 80,57 & 82,99 & 90,34 & 100 & 92,45 & 92,83 & 93,75 & 100,03 \\
\hline 2,5 & 3,85 & 2,66 & 2,43 & 2,97 & 4,86 & 3,57 & 3,03 & 3 , & 49,50 & 51,65 & 57,10 & & 59,41 & 59,48 & 60,64 & 65,48 \\
\hline 3,0 & 1,87 & 1,31 & 1,25 & 1,63 & 2,50 & 1,80 & 1,56 & 1, & 31,16 & 32,70 & 37,14 & 43,42 & 39,24 & 39,55 & 40,10 & 43,71 \\
\hline 4,0 & 0,57 & 0,43 & 0,47 & 0,72 & 0,78 & 0,56 & 0,53 & 0, & 13,24 & 14,12 & 16,63 & & 18,21 & 18,29 & 18,85 & 20,55 \\
\hline 5,0 & 0,22 & 0,20 & 0,29 & 0,53 & 0,28 & 0,23 & 0,30 & 0,53 & 6,17 & 6,63 & 8,11 & 10,54 & 9,25 & 9,29 & 9,64 & 10,53 \\
\hline
\end{tabular}

de controle EWMA. Para grandes deslocamentos $\left(\delta \sigma_{\mathrm{e}}=5,0\right)$, tem-se para o gráfico de $\bar{X}$ um TES $=9,30$ (Tabela 13), enquanto que, para o gráfico de controle de EWMA, o TES = 3,94 (Tabela 14). A conclusão geral que se tira das Tabelas 14 a 18 é que: na presença de um processo com observações da média autocorrelacionadas, a adoção de pequenos valores para $\lambda$ levam aos menores TES, mesmo quando o objetivo é a detecção de grandes deslocamentos da média. Exemplo, para $\phi=0,4, \psi=0,9$ e $\delta=2,0$, o TES se reduz de 59,36 para 7,23 quando $\lambda$ passa de 0,80 (Tabela 17) para 0,05 (Tabela 14). Aplicações em processos autocorrelacionados

Vasconcelos, Alexandre e Freitas (2010) apresentam um processo de fabricação de rolhas metálicas em que cada batida de uma máquina sobre uma chapa metálica produz $k$ rolhas originadas de $k$ diferentes punções. Cada punção equivale a um fluxo de produção e é interesse monitorar o diâmetro médio das rolhas fabricadas. Neste processo, alguns fatores incontroláveis, tais como vibração, desgaste do equipamento e etc., fazem com que o diâmetro médio das rolhas em cada batida oscile e esta oscilação pode ser descrita pelo modelo (2). Além disso, de punção para punção, há uma parcela de ruído ou variação aleatória de rolha para rolha fabricada, cuja média é nula e independe do tempo.

Claro (2008) apresenta um processo de usinagem de uma carcaça fundida em liga de Alumínio. A

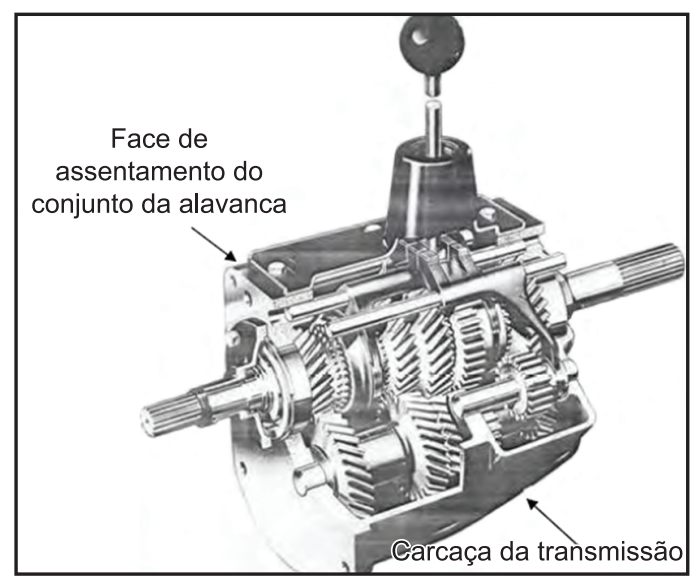

Figura 1. Face de assentamento do conjunto da alavanca.

variável $X$, altura fresada da face de assentamento do conjunto da alavanca (Figura 1), é monitorada e os resultados são apresentados na Figura 2. Os dados da variável em questão foram obtidos de peças consecutivas produzidas em uma indústria da região de Campinas-SP. Das observações de $X$, obtêm-se: média $(\bar{X}=165,02)$, desvio padrão $\left(\sigma_{\mathrm{x}}=0,146\right)$ e valores máximo e mínimo (165,346 e 164,469).

Pode-se considerar que a variabilidade da característica X (altura da face de assentamento) desse processo tem duas parcelas: uma parcela 


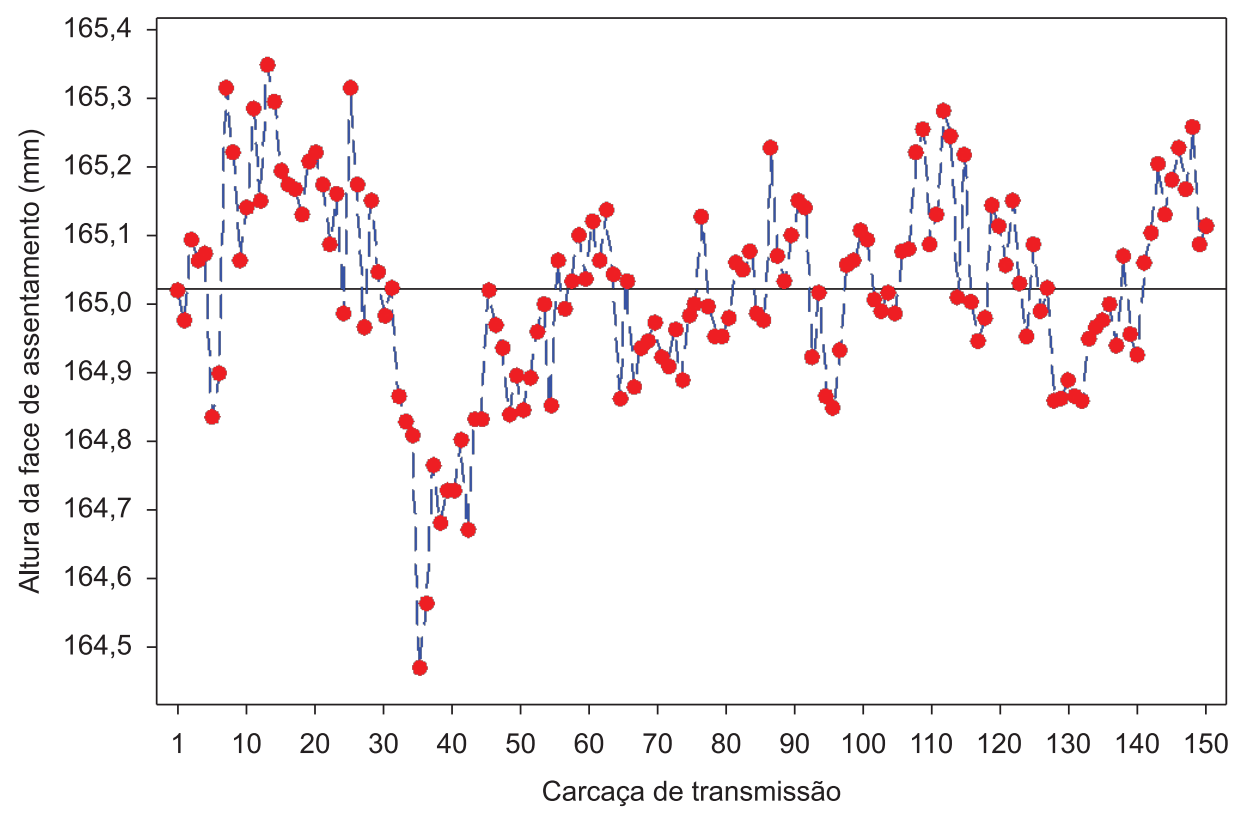

Figura 2. Gráfico das observações das alturas da face de assentamento (mm).

fruto da oscilação aleatória da média do processo segundo um modelo autorregressivo de primeira ordem (variação aleatória de longo prazo) e uma parcela de ruído (variação aleatória de curto prazo) de peça para peça.

O comportamento oscilatório da média é descrito por um AR(1) com parâmetro $\phi=0,894$ (CLARO, 2008). A variabilidade das observações do processo devida à variação em $\mu$ é igual a $\psi=0,79$. A variabilidade total dos dados $\left(\sigma^{2}=0,0214\right)$ pode ser decomposta em duas parcelas: $\sigma_{\mathrm{e}}^{2}=0,004597 \mathrm{e}$ $\sigma^{2} \mu=0,017022$. Para detalhes sobre como obter o valor de $\psi, \sigma^{2} \alpha$ e $\sigma_{\mathrm{e}}^{2}$, veja Reynolds Junior, Arnold e Baik (1996).

Na Tabela 13, há valores de $\phi$ e $\psi$ próximos aos do processo de usinagem. Para deslocamentos de $\delta=0,5$ a $\delta=5,0$, as amostras unitárias levam ao menor TES. Com o esquema EWMA $(\lambda=0,05)$ é preferível utilizar amostras maiores que um, ver Tabela 14 - valores de $\phi$ e $\psi$ próximos aos do processo de usinagem.

\section{Conclusão}

Neste artigo, estudamos o efeito da autocorrelação no planejamento de um gráfico de controle de $\bar{X}$ e de EWMA. Considerou-se que a média do processo oscila em torno de um valor fixo; suas sucessivas observações foram descritas por um modelo autorregressivo de primeira ordem. Um método justo para comparar o desempenho dos gráficos de controle foi proposto e um procedimento bem mais simples para a obtenção do NMA e do TES, envolvendo cadeias de Markov, foi aplicado.
Para utilizar o gráfico de controle de $\bar{X}$, primeiro precisamos determinar seus parâmetros de projeto $(n, h, L)$. Em linhas gerais, se as observações de $X$ são independentes, o procedimento proposto por Costa, Epprecht e Carpinetti (2005) para o planejamento do gráfico de controle de $\bar{X}$ determina que, para pequenos deslocamentos da média, o ideal é trabalhar com amostras grandes, e para grandes deslocamentos, com amostras pequenas.

Contudo, quando a média do processo oscila, e esta oscilação é grande, o ganho em poder de detecção não compensa a adoção de amostras, isto é, o melhor desempenho é alcançado com observações individuais. O mesmo não é observado com o esquema EWMA; se é grande a parcela da variabilidade de $X$ resultante do movimento oscilatório da média do processo, então o mínimo TES é obtido com $\mathrm{n}>1$. Além disso, o TES se reduz significativamente na medida em que diminuímos o valor da constante de amortecimento $\lambda$.

O uso do TES como medida de desempenho pode não ser adequado em algumas situações em que se busca maximizar o uso de recursos ou minimizar as intervenções no processo por questões econômicas. Por exemplo, em processos de estampagem, o objetivo pode ser maximizar o uso das matrizes.

\section{Agradecimentos}

Esta pesquisa contou com apoio da CAPES, do CNPq e da FAPESP. Os autores agradecem aos revisores os valiosos comentários. 


\section{Referências}

CLARO, F. A. E. Gráfico de controle $x$-barra com amostragem dupla para o monitoramento de processos positivamente correlacionados. 2008. Tese (Doutorado em Engenharia Mecânica)-Universidade Estadual Paulista, Guaratinguetá, 2008.

CLARO, F. A. E.; COSTA, A. F. B.; MACHADO, M. A. G. Gráficos de controle de EWMA e de X-barra para monitoramento de processos autocorrelacionados. Produção, v. 17, n. 3, p. 536-546, 2007. http://dx.doi. org/10.1590/S0103-65132007000300010

COSTA, A. F. B.; EPPRECHT, E. K.; CARPINETTI, L. C. R. Controle Estatístico de Qualidade. 2. ed. São Paulo: Atlas, 2005. 334 p.

EPPRECHT, E. K.; SANTOS, A. B. Um método simples para o projeto ótimo de gráficos de X. Gestão \& Produção, v. 5, n. 3, p. 206-220, 1998. http://dx.doi. org/10.1590/S0104-530X1998000300004

LIN, Y. C.; CHOU, C. Y. The variable sampling rate control charts for monitoring autocorrelated processes. Quality and Reliability Engineering International, v. 24, p. 855-870, 2008. http://dx.doi.org/10.1002/qre.927

LIN, Y. C. The variable parameters control charts for monitoring autocorrelated processes. Communications in Statistics- Simulation and Computation, v. 38, p. 729-749, 2009. http://dx.doi. org/10.1080/03610910802645339

LU, C. W.; REYNOLDS JUNIOR, M. R. EWMA control charts for monitoring the mean of autocorrelated processes. Journal of Quality Technology, v. 31, p. 166-188, 1999a.

LU, C. W.; REYNOLDS JUNIOR, M. R. Control chart for monitoring the mean and variance of autocorrelated processes. Journal of Quality Technology, v. 31, p. 259-274, 1999 b.

LU, C. W.; REYNOLDS JUNIOR, M. R. Cusum charts for monitoring an autocorrelated process. Journal of Quality Technology, v. 33, p. 316-334, 2001.

REYNOLDS JUNIOR, M. R.; ARNOLD, J. C.; BAIK, J. W. Variable sampling interval charts in the presence of correlation. Journal of Quality Technology, v. 28, p. 12-30, 1996.

SHEWHART, W. A. Economic Control of Quality of Manufactured Product. New York: D.Van Nostrand Company, 1931. 501 p. Republicado em 1980 por American Society for Quality, Chelsea-MI-EUA.

VAN BRACKLE III, L. N.; REYNOLDS JUNIOR, M. R. EWMA and CUSUM control charts in the presence of correlation. Communication in Statistics - Simulation and Computation, v. 26, p. 979-1008, 1997.

VASCONCELOS, A. P.; ALEXANDRE, J. W. C.; FREITAS, S. M. Um estudo sobre o desempenho e eficiência do gráfico de controle por grupos em processos paralelos. In: ENCONTRO NACIONAL DE ENGENHARIA DE PRODUÇÃO - ENEGEP, 30., 2010, São Carlos. Anais... São Carlos, 2010.

ZOU, C.; WANG, Z.; TSUNG, F. Monitoring autocorrelated process using variable sampling schemes at fixed-times. Quality and Reliability Engineering International, v. 24, n. 1, p. 55-69, 2008. http://dx.doi.org/10.1002/qre.867 


\section{Apêndice 1. Determinação do NMA como Função do Número Esperado de Visitas aos Estados Transientes de uma Cadeia de Markov.}

Como a média $\mu$ do processo oscila de acordo com um modelo AR (1), utilizamos o seu valor a cada instante de amostragem para definir os estados transientes da cadeia de Markov. Para trabalhar com uma cadeia de Markov finita, discretizamos $\mu$ em m m valores $\mu_{1}, \mu_{2}, \ldots, \mu_{\mathrm{m}}$. Se o valor de $\bar{X}$ da amostra (i) pertence à região central do gráfico de controle, então o valor $\mu(i+1)$ da média do processo, quando a amostra $(i+1)$ é formada, define o estado transiente da cadeia de Markov. Se $\mu(i+1)=\mu_{1}$, o estado $(l)$ é alcançado, em que $l \in\{1,2, \ldots, m\}$. O estado absorvente é alcançado quando o gráfico de controle sinaliza um desajuste do processo, isto é, quando um valor de $\bar{X}$ surgir na região de ação. A matriz de probabilidades de transição é dada por

$$
\mathrm{Q}=[\mathrm{p}(r, s)], \quad r, \mathrm{~s} \in\{1,2, \ldots, m\}
$$

em que $p(r, s)$ representa a probabilidade de transição do estado $(r)$ para o estado $(s)$. Se a média do processo é discretizada em m m valores $\mu_{1}, \mu_{2}, \ldots, \mu_{\mathrm{m}}$, com $\mu_{j}=\mu_{0}-[(m-1)-2(j-1)] \Delta$ e $\Delta=7 \sigma_{\mu}(m-1)^{-1}$, segue então

$$
p(r, s)=\operatorname{Pr}\left\{\left|\bar{X}-\mu_{0}\right|<L \sigma_{\bar{X}} \mid \bar{X} \sim N\left(\mu_{r} ; \frac{\sigma_{e}^{2}}{n}\right)\right\} \operatorname{Pr}\left\{\left|\alpha-\mu_{s}+\phi^{h} \mu_{r}\right|<\Delta \mid \alpha \sim \mathrm{N}\left[0 ;\left(1-\phi^{2 h}\right) \sigma_{\mu}^{2}\right]\right\}
$$

O número médio de amostras até o sinal é então dado por

$$
\mathrm{NMA}=b^{\prime}(\mathrm{I}-\mathrm{Q})^{-1} t
$$

sendo $b=\left(p_{1}, p_{2}, \ldots, p_{m}\right)$ com $p_{i}=\operatorname{Pr}\left\{\left|\mu-\mu_{\mathrm{i}}\right|<\Delta \mid \mu \sim N\left(\mu_{0} ; \sigma_{\mu}^{2}\right\}\right.$ o vetor de probabilidades iniciais e $t^{\prime}=(1,1, \ldots, 1)$ de intervalos entre amostragens, respectivamente. 